\title{
POTENCIALIDAD DEL CULTIVO EN BALSA DE Semimytilus algosus (GOULD) 1850, EN LA BAHIA DE CONCEPCION (MOLLUSCA, BIVALVIA, MYTILIDAE)
}

\author{
JOSE GONZÁLEZ, JORGE VILLAGRA, MAURICIO HINOJOSA Y RAÚL BECERRA \\ Pontifícia Universidad Católica de Chile, Sede Regional Talcahuano, \\ Departamento de Biología y Tecnología del Mar
}

\section{SYNOPSIS}

\begin{abstract}
Semimytilus algosus (Gould) 1850 , constitutes a potencial resource for cultivation in Chile. The geographic distribution exrenas from Manta, Ecuador to the Gulf of Arauco in Chile (Osório \& Bahamonde, 1968). Juveniles with a $7 \mathrm{~mm}$ mode were collected from the mouth of the B to-B to River $\left(36^{\circ} 48^{\prime} \mathrm{S}, 73^{\circ} 10^{\prime} \mathrm{W}\right)$, and were encorded using the Spanish and French systems. These cords, $3 \mathrm{~m}$ long, were suspended from an experimental raft $(4 \times 4 \mathrm{~m})$ in the western part of Concepcion Bay $\left(36^{\circ} 42^{\prime} S, 72^{\circ} 02^{\prime} \mathrm{W}\right)$. After $11 \mathrm{months}$ of suspension the mussels had attained a modal lenght of $52 \mathrm{~mm}$, and absolute increase of $45 \mathrm{~mm}$. The maximun length measured was $67 \mathrm{~mm}$. A parallel study of the natural population revealed a slow growth rate, with much smaller maxima than those obtained with the raft. The experimental population seemed to exhibit a short life cycle, as demostrated in part by the rapid growth rate and a high natural death rate before completing a year of suspension. A histological analysis showed S. algosus to be hermafroditic, with mature gametes during the entire year. The gametes varied only slightly in shape and size from the gametes described for other mussel species. Canning tests were made in the local industries. Individuals with a modal length of $36.38 \mathrm{~mm}$ yielded $30 \%$, while those of $30 \mathrm{~mm}$ individuals used in this experiment, S. algosus demonstrated a yield that compared favorably with that of other comercially important mussels, and would seem to present a great potential for a future indus. trial resource.
\end{abstract}

\section{Introduccion}

Como consecuencia del deterioro de los suelos agrícolas del mundo, surge el mar como posibilidad cierta de fuente alimenticia. Ello, baseado en la innegable riqueza de éste el que, por desconocimiento o negligencia, se ha ido explotando en exceso. Así, se hace necesario que cada día más gente conozca el significado del cuidado en la explotación de las especies marinas, aprenda a regular su extracción y a fomentar el consumo de otras especies que, teniendo un futuro comercial, aún no han sido explotadas.

Castilla \& Becerra (1976) señalan que, en Chile existen más de 60 especies de mariscos, de los cuales $30-40$ son importantes desde un punto de vista comercial.

Entre los moluscos de las costas chilenas, hay casos criticos de especies en inminente vía de extinción, como el choro zapato, Choromytilus chorus (Molina) 1782, y el ostión, Argopecten purpuratus (Lamarck) 1819; otros gravemente sobreexplotados, como la cholga, Aulacomya ater (Molina) 1782, el loco, Concholepas concholemas Bruguiere 1789. Además, se ha sometido a una intensa explotación a un equinoderno, el erizo blanco, Loxechinus albus Molina 1782.

Con estos antecedentes se abren dos perspectivas, las que pueden ser desarrolladas simultáneamente: una es el cultivo artificial de las éspecies anteriores, y la otra, es estudiar algunas especies que suplan la falta de las anteriormente nombradas. Tratando de unificar estas perspectivas, se ha comenzado un estudio sobre la biología del chorito negro, Semimytilus algosus (Gould) 1850 , en cultivo suspendido en balsa y de fondo, en la Bahía de Concepción.

Osorio \& Bahamonde (1968) describen para esta especie, una talla máxima de $54 \mathrm{~mm}$ em banco natural, y su distribución geográfica desde Manta, Ecuador, hasta el Golfo de Arauco en Chile.

Tomicic (1968) señala que esta especie alcanza $60 \mathrm{~mm}$ en los muelles de la ciudad de Mejillones, y $80 \mathrm{~mm}$ aquellos que se fijan a las cuerdas de cultivo de cholgas.

Gonzalez (en prensa), controló una talla máxima individual de $99 \mathrm{~mm}$, en cuerdas suspendidas en Arica.

Basado en lo anterior, se hace necesario conocer la dinámica poblacional de la especie, en aspectos relacionados con crecimiento, reclutamiento y mortalidad, así como determinar el ciclo biológico de $S$. algosus, con especial énfasis en el desarrollo sexual. La prioridad del estudio de los ciclos gonádicos y madurez sexual de esta especie, se basa en la necesidad de obtener captación de juveniles, ubicando oportunamente los colectores para un eventual programa de desarrollo de cultivo.

Teniendo en cuenta que los estudios relacionados con la biología de los mitílidos se basan principalmente en los géneros Mytilus, Aulacomya y Choromytilus, se estima que un estúdio sobre la biología básica de $S$. algosus puede servir de aporte al conocimiento de esta familia de moluscos.

La mayoría de las investigaciones sobre mitílidos están orientados a los campos de la dinámica poblacional, taxonomía y distribución.

El aspecto de sexualidad y madurez sexual es mencionado, entre otros, por Stuardo (1962); Tomicic (1966); Lozada (1968); Saelzer (1969); Lozada et al. (1971 y 1974); Solis \& Lozada (1971); Padilla (1973); Aracena et al. (1974) y Carreño (1977).

\section{Resultados y discusion}

Hermafroditismo: En general los mitflidos son especies dioicas. Lunetta (1969, fide Lubet 1959) señala que el hermafroditismo en mitílidos es muy raro, no alcanzando a $0.1 \%$ en especies del género Mytilus. froditismo.

$S$. algosus es una de las excepciones que presenta herma-

Sistema reproductor: Formado por varios conductos ramificados que parten desde el mesosoma (Chipperfield, 1953). En períodos de máxima madurez, estos ocupan casi todo el manto. En esse instante, las gónadas presentan un color amarillo intenso en el sector macho y café oscuro en el lado hembra.

Se demostró que las gónadas reaccionan de diferente manera a la tinción corriente. Los folículos masculinos tienen un comportamiento eosinófilo, en tanto que los femeninos, uno basófilo.

Los gametos maduros son vaciados por dos conductos genitales, encontrándose uno a cada lado del cuerpo, próximos a las lamelas branquiales.

$S$. algosus madura con ambas gónadas en forma paralela, lo que permitiría postular que, podría existir autofecundación externa.

Descripción de las células sexuales:

Células femeninas: Predominan las formas poliédricas, esferoidales y piriformes.

Ovogonias: De forma muy irregular y adosadas a la pared del folículo. E1 tamaño promedio es de 18.5 micras.

Ovocitos en previtelogénesis: Se observan dos tipos de células, las más pequeñas con un promedio de 28.5 micras de diámetro máximo. Son de forma achatana y adosados a la parede folicular.

Las mayores, con un promedio de 53.5 micras y también se encuentran adheridas a la pared del folículo.

Ovocitos en vitelogénesis: De forma variable, aunque tienden a ser esféricas. Se observan libres en el centro del folículo. El tamaño medio es de 61.5 micras.

Ovulos maduros emitidos: Células que ya han salido del cuerpo del animal. Forma esférica. Diámetro promedio, 66 micras.

La forma generalmente poliédriea de las células femeninas, puede deberse a la presión que ejercen las células vecinas, o bién, por efectos de deshidratación.

Células masculinas: Ocupan una mayor parte del cuerpo del animal, que los folículos femeninos.

Espermatogonias: Generalmente de forma esférica y adosadas a la pared folicular. Su tamaño fluctúa entre 5.5 y 6.0 micras.

Espermatocitos I: De forma generalmente esférica. Se encuentran a continuación de las espermatogonias.

Espermatocitos II: Esféricos: se encuentran entre los espermatocitos I y las espermátidas. Tamaño promedio: 2.3 a 3.0 micras.

Espermátidas: De forma cónica. Se observan entre los espermatocitos II y los espermios. Tamaño promedio: 3.0 micras.

Espermios: Se encuentran en el centro del folículo. Sen cónicos, con la región anterior muy aguzada, Longitud promedio de la cabeza, 6.2 micras. Diámetro maximo de la cabeza, 2.2 micras. Las formas de las células germinales encontradas en $S$. algo. sus, no difieren mayormente a las descritas por Carreño (1977) para 


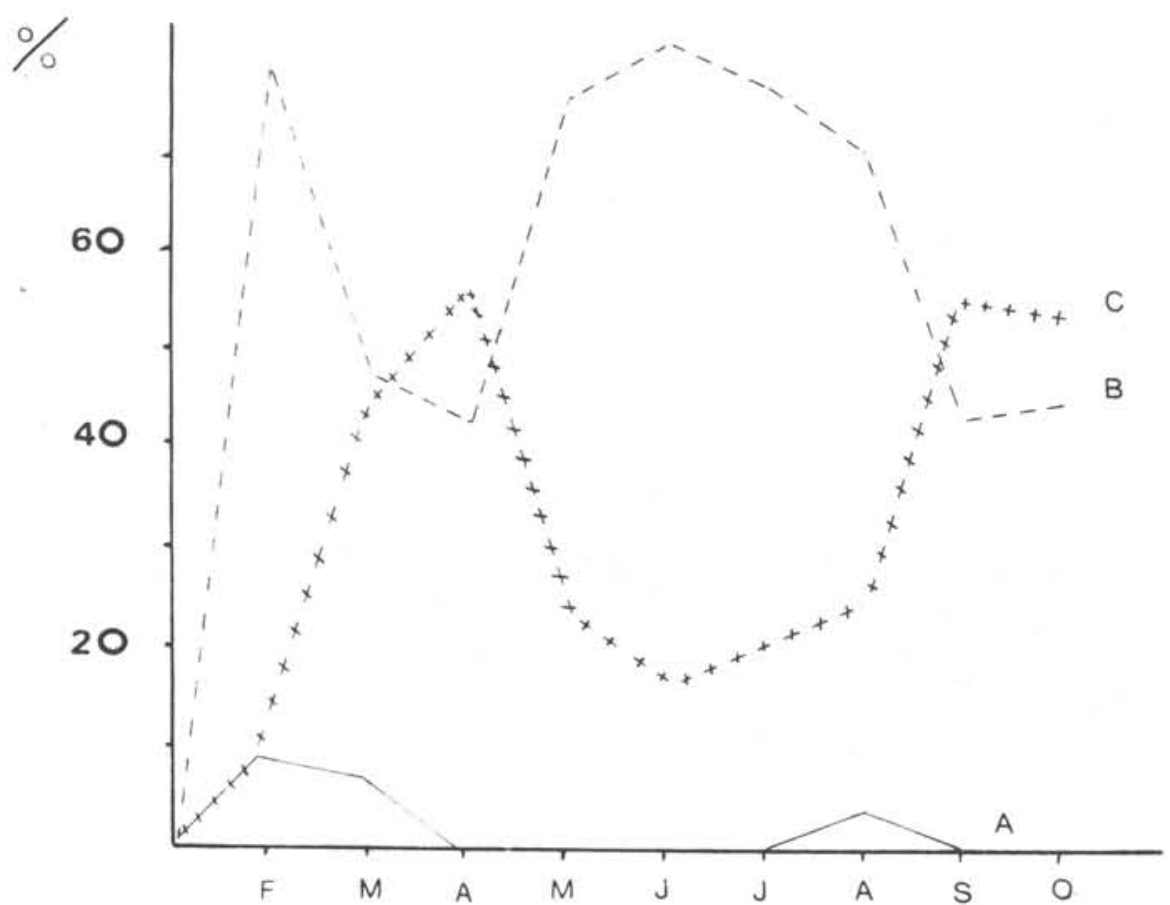

Fig. 1 -Variación mensual de los estados de madurez. $\mathrm{A}=$ inmaduros; $\mathrm{B}=$ en maduración y maduros; $\mathrm{C}=$ en desove y desovados.
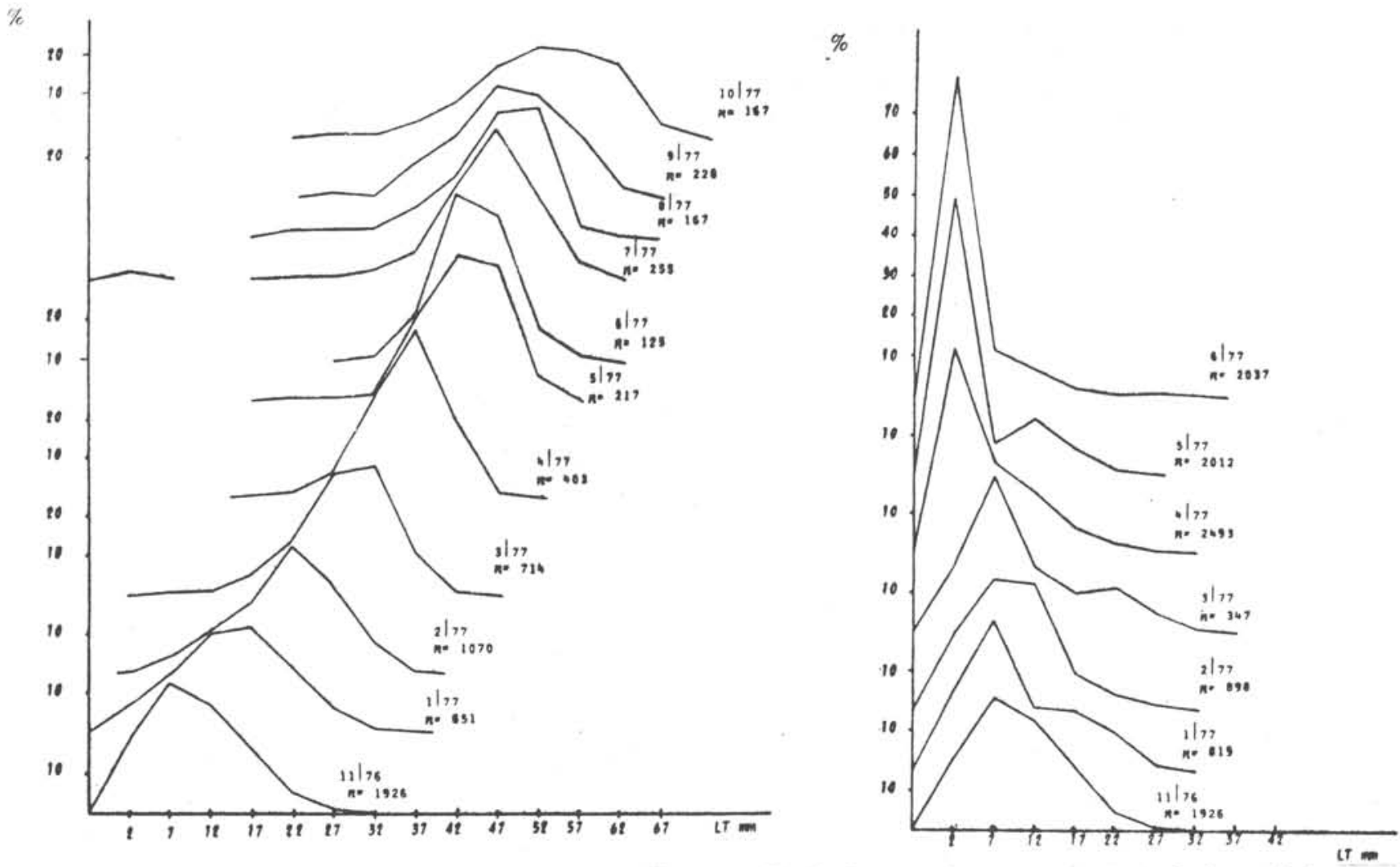

Fig. 2 -Composición de la población de $S$. algosus de cultivo en los diferentes meses muestreados.

Fig. 3 -Composición de la población de $S$. algosus de banco natural, en los diferentes meses muestrados. 
Aulacomya ater. Una diferencia se encuentra en las espermátidas que, según Carreño (op. cit.) son "desde alargadas a esféricas", y en $S$. algosus son claramente cónicas.

El diámetro promedio de los óvulos es de 66 micras y el de espermios es de 2.2 micras, siendo su longitud de 6.25 micras, valores cercanos a los encontrados por Lozada et al. (1971) para Ch. chorus, y por Carreño (op. cit.) para A. ater.

Estados de madurez sexual: Chipperfield (1953) señala una escala de cuatro estados pare Mytilus edulis. En S. algosus se detectó dos de los cuatro estados descritos por Chipperfield (op. cit). Gónada en estado II: Folículos bién desarrolados, pero sin alcanzar la madurez sexual.

Gónada en estado III: Gónadas maduras y listas para la emisión. $\mathrm{La}$ ausencia de individuos en estados de madurez 0 y 1 , según la escala de Chipperfield (op. cit.), puede deberse a que sólo se trabajó con ejemplares mayores de $7 \mathrm{~mm}$, los que ya presentaban un estado II. Con ello, se podría suponer que esos estados, se darían en mayor proporción en individuos de $0-6 \mathrm{~mm}$.

Ciclo de madurez sexual: Se utilizo la escala de madurez sexua establecida por Lozada (1968) y simplificada por Solis \& Lozada (1971), que agrupa los ejemplares en tres estados: $\mathrm{A}=$ inmaduros; $\mathrm{B}=$ en maduración y maduros; $\mathrm{C}=$ en desove $\mathrm{y}$ desovados. (Fig. 1).

Los individuos en estado A (Fig. 1), son juveniles encontrados en los bisos de los adultos. Presentaban una talla inferior a $2 \mathrm{~mm}$ y examinados bajo lupa, no mostraban trazas de sexualidad.

Hernandez \& Gonzalez (1976) señalan que, para Mytilus chilensis de cultivo suspendido, se observa dos épocas de desove, una en invierno-primavera y otra en verano-otoño. Con algunas variaciones, estos piques coinciden con los detectados en $S$. algosus, aunque esta diferencia puede estar afectada, por la distribución latitudinal de las especies (Wilson \& Hodkin, 1967).

Crescimiento en balsa: En noviemore de 1976, los ejemplares tenian una moda única de $7 \mathrm{~mm}, \mathrm{y}$ al cabo de 11 meses presentaron una moda máxima de $52 \mathrm{~mm}$, lo que significó un incremento de $45 \mathrm{~mm}$ (Fig, 2).

En enero de 1977. los ejemplares de segmentos superiores presentaron una moda de $17 \mathrm{~mm}$, en tanto que los de segmentos inferiores tenían una moda de $12 \mathrm{~mm}$. En mayo del mismo año se observó modas de 42 y $32 \mathrm{~mm}$ respectivamente.

En junio de 1977, los ejemplares de los niveles superiores, tanto en cuerdas francesas y españolas, presentaban una moda de $42 \mathrm{~mm}$. Los choritos de cuerdas francesas de los niveles inferiores, tenían una moda de $27 \mathrm{~mm}$. de $67 \mathrm{~mm}$

En octubre de 1977 , se controló una talla máxima individual

Crecimiento en banco natural: En noviembre de 1976, los ejemplares presentaban una moda de $7 \mathrm{~mm}$, la que se repitió en los meses de enero, febrero y marzo, en tanto que en abril, mayo y junio del mismo año, se observa una moda de $2 \mathrm{~mm}$ (Fig. 3). A pesar de mantenerse las modas, se observa leves variaciones en el porcentaje de ejemplares de las marcas de clase mayores de $7 \mathrm{~mm}$. Según esto, se detecta un rápido crecimiento de los ejemplares, sin embargo, las longitudes no alcanzan los valores de los ejemplares cultivados. La talla máxima individual controlada fue de $38 \mathrm{~mm}$.

Reclutamiento en balsa: Se detectó reclutamiento en el mes de julio de 1977, en un $1.96 \%$ del total de la muestra (Fig. 2); estos ejemplares se observaron en los bisos de los reproductores.

En los colectores experimentales instalados en la periferia de la balsa, prácticamente no se detectó presencia de juveniles, a excepción del mes de junio en que se encontró 60 ejemplares de $S$. algosus, cuyas tallas oscilaron entre $0-4 \mathrm{~mm}$, presentes en el primer metro superficial.

Mortalidad en balsa: En enero se observa una mortalidad de $21,81 \%$, la que en febrero, marzo, abril, mayo y junio se mantiene relativamente constante con un valor medio de $5.05 \%$. Finalmente, en julio la mortalidad es de $10.98 \%$, y en los meses seguientes continúa aumentando hasta llegar a noviembre, en que ella es de $100 \%$.

Mortalidad en banco natural: La mortalidad observada en ejemplares de banco natural es muy baja, detectándose un valor máximo en noviembre de 1976 con un $3.22 \%$ en ejemplares de $177 \mathrm{~mm}$ y un mínimo en mayo con un $0.54 \%$ en ejemplares de $11 \mathrm{~mm}$.

Rendimiento y envasado del producto: En rendimiento en carne obtenido de ejemplares cultivados fue de $30 \%$ en choritos de $37 \mathrm{~mm}$ de moda, y de $40 \%$ en aquellos de $30 \mathrm{~mm}$ de moda, a diferencia de lo observado en ejemplares silvestres en los que se detectó un $17 \%$ de rendimiento en ejemplares de $27 \mathrm{~mm}$.
Se fabrico tres tupos de productos: chorito al aceite, pate de choritos y paté de choritos con jurel (proporción 1:1). En un panel de degustación, se apreció la aceptación del producto elaborado.

\section{Conclusiones}

S. algosus es mayoritariamente hermafrodita, presentándose escaso número de individuos con un solo sexo.

Los gametos no difieren mayormente, en forma y tamaño, a los descritos para otras especies de mitílidos.

Ambas gónadas maduran en forma paralela y los gametos son vaciados por un conducto genital par.

$S$, algosus presenta gametos maduros durante todo el año, observándose dos piques máximos de desove.

En 11 meses de suspensión se observó un incremento absoluto de $45 \mathrm{~mm}$.

La talla máxima controlada en cultivo fue de $67 \mathrm{~mm}$ y de $38 \mathrm{~mm}$ en banco natural.

La mortalidad observada en balsa fue de un $100 \%$, después de 11 meses y de $3.22 \%$ la máxima controlada en banco natural El rendimiento industrial en carne de los ejemplares oscilo entre $30-40 \%$. En los de banco natural fue de $17 \%$.

\section{Referencias Bibliográficas}

ARACENA, O.; YAÑEZ, R.; LOZADA, E. \& LOPEZ, M.T. 1974 Crecimiento de Choromytilus chorus en Talcán, Chiloé (Mollusca, Bivalvia, Mytilidae). Boln. Soc. Biol. Concepción, (48) : 347-357.

CARREÑO, A. 1977. Gametogénesis y ciclo de madurez sexual en Aulacomya ater. Tésis. Fac. Cienc. Univ. de Chile, Santiago. (mimeografiada).

CASTILLA, J.C. \& BECERRA, R. 1976. The shellfisheries of Chile: an analysis of the statistics 1960-1973. Proc. Symp. Upwelling, (Univ. del Norte-Fundación Chile). Santiago, Editorial Universitaria, p. 61-90.

CHIPPERFIELD, P. N. I. 1953. Observations on the breeding and settlement of Mytilus edulis (L) in British waters. J. mar. biol. Ass. U.K., $32: 449-476$.

GONZẢLEZ, J. Consideraciones generales sobre el crecimiento en balsa del chorito negro, Semimytilus algosus (Gould) 1850, en Arica, Chile. Boln Soc. Biol. Concepción, (51) (en prensa).

HERNÁNDEZ, J. \& GONZẢLEZ, L. 1976, Observaciones sobre el comportamiento de mitílidos chilenos en cultivo suspendido. I. Chorito (Mytilus chilensis, Hupe, 1854). Ser. Investigación pesq., Inst. Fomento pesq., (22) : 1-52.

LOZADA. E. 1968, Contribución al estudio de la cholga, Aulaco. mya ater en Putemún. Biol. pesq. Chile, (3):3-39.

- - ; HERNẢNDEZ, J.M.; ARACENA, O. \& LOPEZ, M.T 1974. El cultivo de la cholga (Aulacomya ater) en Isletilla Estereo de Castro, (Mollusca, Bilvavia, Mytilidae). Boln Soc. Biol. Concepción, (48) : 331-346.

---; ROLLERI, J. \& YANẼZ, R. 1971. Consideraciones biológicas de Choromytilus chorus en dos sustratos diferentes. Biol. pesq. Chile, (5):61-108.

LUNETTA, J. E. 1969. Fisiologia da reprodução dos mexilhões (Mytilus perna). Bolm Fac. Filos. Ciênc. Letr. Univ. S. Paulo, (324), Zool. Biol. mar., n.s., (26) : 33-115.

OSORIO, C. \& BAHAMONDE, N. 1968. Moluscos bivalvos en pesquerías chilenas. Biol. pesq. Chile, (3):69-128.

PADILLA, M. 1973. Observaciones biológicas relacionadas con el cultivo de Mytilus edulis chilensis en Aysén. Publnes Inst. Fomento pesq., (54) : 1-23.

SAELZER, H. 1969. Reconocimiento a los bancos de mitílidos en la zona de Castro (Chiloé). Boln Soc. Biol. Concepción, (16) : 135-153.

SOLIS. I. \& LOZADA, E. 1971. Algunos aspectos biológicos de la cholga de Magallanes (Aulacomya ater MoL.). Biol. pesq. Chile, (5) . 109-144

STUARDO, J. 1962. Informe de los estudios sobre mitilicultura realizados hasta septiembre de 1961. Investnes cient. Dep. Pesca Caza, Santiago, I, : 1-27 + fig.

TOMICIC, J. 1966. Contribución al estudio de la cholga Aulaco. mya ater (Molina) en la bahía de Mejillones. Memoria de Prueba. Universidade de Chile, Antofagasta, 19 p. fig.

- . - 1968. Una especie con futuro comercial (chorito negro). Apunt, oceanol., (4) : 16-17.

WILSON, B.R. \& HODKIN, E. 1967. A comparative account of the reproductive cycles of five species of marine mussels (Bivalvia, Mytilidae) en the vicinity of Freemantle, Western Australia. Aust. J. mar. Freshwat. Res., 18:175-203. 